\title{
Valoración de ansiedad y burnout en los profesionales de enfermería de un servicio de Nefrología
}

\author{
Ángel Jiménez Maldonado, Sonsoles García Millán \\ Servicio de Nefrología. Hospital Universitario del Río Hortega. Valladolid. España
}

\section{Resumen}

Introducción: La enfermería es por naturaleza una profesión con alto riesgo de generar estrés entre sus profesionales. Diversos estudios así lo demuestran ${ }^{1,2 .}$

\section{Objetivos:}

- Valorar la presencia del burn out en nuestro servicio.

- Valorar si existen diferencias entre TCAE y enfermeras.

- Valorar si hay diferencias según la edad y el tiempo en el servicio.

\section{Metodología:}

Se realizó un estudio de tipo cualitativo, descriptivo, transversal y monocéntrico, en el servicio de nefrología del Hospital U. Rio Hortega de Valladolid.

Para ello se utilizó dos cuestionarios, el BMI y el STAI.

\section{Resultados:}

Completaron los cuestionarios el 77,27 \%. La muestra fue 11 enfermeras y 5 TCAE y solo uno era un hombre. Las edades oscilan de 28 y 62 años y el tiempo medio en el servicio fue 11,1 años.

\section{TEST MBI:}

- Subescala cansancio emocional: El $12.5 \%$ niveles muy altos, el $6.25 \%$ niveles medios y el resto niveles bajos.

- Subescala de despersonalización: El 12.5\% tenía niveles muy altos, el $25 \%$ niveles medios y el $62.5 \%$ niveles bajos.

\section{Correspondencia: \\ Ángel Jiménez Maldonado \\ C/ Villanueva de los infantes $n^{\circ} 27$ \\ Renedo de Esgueva. 47170 Valladolid \\ E-mail: jijasan@gmail.com}

- Subescala de realización personal: El 25\% tenía niveles bajos, el $31.25 \%$ tenía niveles medios y el $44.75 \%$ tenía niveles altos.

Tomando como variables el tiempo trabajado y la edad no hay diferencia estadísticamente significativa.

La media para la escala STAI-S fue de $19.53 \pm 3.02$ y para STAI-T fue de 14,24 $\pm 7,12$.

\section{Conclusiones:}

Los profesionales sanitarios evaluados por el BMI tienen bajos niveles de agotamiento, y de ellos sólo una persona tiene síndrome de burn out. Las enfermeras tienen más agotamiento emocional pero están más satisfechas que las TCAE y la ansiedad es de carácter predominantemente transitorio en ambas.

PALABRAS CLAVE

- ANSIEDAD

- BURNOUT

- ENFERMERÍA NEFROLÓGICA

Assessment of anxiety and burnout in nurses of a unit of Nephrology

\section{Abstract}

Introduction: Nursing is by nature a profession with high risk of generating stress among professionals. Several studies show it.

\section{Objectives:}

- Assess the presence of burn out in our unit.

- Assess whether there are differences between nursing assistants and nurses.

- Assess whether there are differences according to age and time of service. 


\section{Methodology:}

A qualitative study, descriptive, cross-sectional and single-center, in the nephrology unit of the University Hospital Rio Hortega of Valladolid was carried out. For this purpose two questionnaires, BMI and STAI were used.

\section{Results:}

Questionnaires were completed on $77.27 \%$. The sample was 11 nurses and five nursing assistants and only one was a man. The ages range from 28 to 62 years and the average time the unit was 11.1 years.

\section{TEST MBI:}

- Emotional exhaustion subscale: $12.5 \%$ very high levels, the $6.25 \%$ average levels and the rest low levels.

- Depersonalization subscale: $12.5 \%$ had very high levels, $25 \%$ average and $62.5 \%$ low levels.

- Personal fulfillment subscale: $25 \%$ had low levels, $31.25 \%$ had average levels and $44.75 \%$ had high levels.

Taking as variables, time worked and age there are no statistically significant differences. The average for the STAI-S scale was $19.53 \pm 3.02$ and STAI-T was 14.24 \pm 7.12 .

\section{Conclusions:}

Healthcare professionals evaluated by BMI have low levels of exhaustion, of which only one person has a burn out syndrome. Nurses have more emotional exhaustion but are more satisfied than nursing assistants, and anxiety in both professions is predominantly transient.

\section{KEYWORDS}

- ANXIETY

- BURNOUT

- NEPHROLOGY NURSING

\section{Introducción}

El término "Burn out" procede del inglés y se traduce en castellano por "estar quemado" .

En 1976, la psicóloga Cristina Maslach4 utilizó el término Burn out, y lo definió como "síndrome de ago- tamiento emocional, despersonalización y baja realización personal que puede ocurrir entre individuos cuyo trabajo implica atención o ayuda a personas".

Maslach $^{4}$ y Jackson, entienden que está configurado como un síndrome tridimensional caracterizado por:

- El agotamiento emocional y físico que se caracteriza por una ausencia o falta de energía, entusiasmo y un sentimiento de escasez de recursos. A estos sentimientos pueden sumarse los de frustración y tensión en los trabajadores que se dan cuenta que ya no tienen condiciones de gastar más energía.

- La despersonalización o deshumanización que se caracteriza por tratar a los clientes, compañeros y la organización como objetos. Los trabajadores pueden demostrar insensibilidad emocional, un estado psíquico en que prevalece el cinismo o la disimulación afectiva, la crítica exacerbada de todo su ambiente y de todos los demás.

- La disminución de la realización personal en el trabajo que se caracteriza como una tendencia del trabajador a autoevaluarse de forma negativa. Las personas se sienten infelices consigo mismas, insatisfechas con su desarrollo profesional, experimentan una declinación en el sentimiento de competencia y de éxito en su trabajo y en su capacidad de interactuar con las personas.

Para diagnosticar un síndrome de Burn out se suele aplicar principalmente el test $\mathrm{MBI}^{5}$ (Maslach Burnout Inventory), que valora las tres dimensiones anteriormente citadas (el agotamiento personal, la despersonalización y la realización personal). Se consideran que las puntuaciones en cada apartado del MBI son bajas entre 1 y 33 y que puntuaciones altas en los dos primeros y baja en el tercero definen el síndrome.

La enfermería al estar en contacto directo con personas que tienen problemas de salud y con sus familiares, es una de las profesiones en las que más estudios sobre el Síndrome de Burn out se realizan 5 .

Hay que tener en cuenta que la enfermería nefrológica, a diferencia de otras especialidades de enfermería, se desarrolla fundamentalmente en la atención al paciente crónico durante un periodo de tiempo más o menos prolongado, en el cual se suelen establecer lazos afectivos con el paciente y su familia. Esto puede suponer, ante los cambios que experimenta el paciente en su estado de salud a lo largo del tiempo, un plus de sobrecarga emocional de los profesionales de enfermería. 
Además, desde otro punto de vista, la enfermería nefrológica se mueve en un entorno muy especializado en el que los cambios tecnológicos están a la orden del día. Esto implica una exigencia a los profesionales para su actualización, pudiendo esto suponer, que el personal de enfermería nefrológica ${ }^{6}$ suela estar sometido a situaciones de estrés que pueden derivar en situaciones de ansiedad al tener que asumir estos cambios.

Por tanto, nos planteamos como objetivos, valorar los niveles de ansiedad y detectar la presencia del burn out en los profesionales de enfermería de nuestro servicio, valorando si existen diferencias entre técnicos en cuidado de enfermería (TCAE) y enfermeras, y valorar si hay diferencias según la edad y el tiempo en el servicio.

\section{Material y Método}

Presentamos un estudio de tipo cualitativo, descriptivo, transversal, cuya población abarcó a los profesionales de enfermería del servicio de Nefrología del Hospital Universitario Rio Hortega de Valladolid, que desempeñan su actividad en unidades de hemodiálisis, consulta ERCA y prediálisis, y que voluntariamente quisieran someterse a valoración.

Se llevó a cabo durante noviembre y diciembre del año 2014.

Los participantes fueron evaluados en el lugar de trabajo y se les sugirió que cumplimentaran los cuestionarios en lugares tranquilos, tratando de mantener ciertas condiciones de intimidad. Además, se les informó a cada uno de ellos sobre los objetivos del estudio, así como también de la condición de anonimato bajo la cual se iban a tratar los datos.

A cada participante se le pasó el Inventario de Ansiedad Estado-Rasgo (STAI) ${ }^{7}$ y el Maslach Burnout Inventory (MBI), así como un registro en el que se recogían las siguientes variables: edad, sexo, estado civil, tiempo trabajando en la unidad y categoría profesional.

EI STAI, en ingles State-Trait Anxiety Inventory, fue creado por Spielberger, Gorsuch y Lushene en $1970^{8}$.

Es un inventario autoadministrado que evalúa dos conceptos independientes de la ansiedad: la ansiedad como estado y la ansiedad como rasgo.

Para ello consta de dos subescalas de 20 ítems cada una:
- La subescala Ansiedad-Estado (STAI-S) evalúa un estado emocional transitorio, caracterizado por sentimientos subjetivos, conscientemente percibidos, de atención y aprensión y por hiperactividad del sistema nervioso autónomo. En esta parte el sujeto indicará en qué medida cada enunciado refleja como se siente "ahora mismo, en este momento", mediante una escala de 1 a 4: "Nada"; "Algo"; "Bastante" y "Mucho".

- La subescala Ansiedad-Rasgo (STAI-T) valora una propensión ansiosa, relativamente estable, que caracteriza a los individuos con tendencia a percibir las situaciones como amenazadoras. El marco de referencia temporal en este caso de la ansiedad como rasgo es "en general, habitualmente". La escala de respuestas para las frases de esta escala también es de tipo Likert y sus opciones son: "Casi nunca"; "A veces"; "A menudo" y "Casi siempre".

El tiempo de aplicación total ronda los 20 minutos aproximadamente.

Brinda una puntuación de ansiedad como estado y otra de ansiedad como rasgo, cada una de las cuales se obtiene sumando las puntuaciones en cada uno de los 20 ítems correspondientes. En cada una de las escalas, encontramos ítems que denotan la presencia o la ausencia de ansiedad.

En los ítems que indican la presencia de ansiedad se evaluará: 1 - 2 - 3 - 4; en los ítems que denotan la ausencia de ansiedad se evaluará a la inversa: 4 - 3 - 2 - 1 .

La puntuación total para cada una de las subescalas oscila entre 20 y 80 puntos en la versión inglesa. Sin embargo en este caso nosotros usamos la versión española que oscila entre 0 y 60 puntos, ya que en la escala española se evaluará la presencia de ansiedad: 0 - 1 - 2 - 3; los ítems que denotan la ausencia de ansiedad se evaluará a la inversa: 3 - 2 - 1 - 0.

No existen puntos de corte propuestos, sino que las puntuaciones directas que se obtienen se transforman en centiles en función del sexo y la edad.

Los centiles, representan medidas de posición en una distribución de frecuencias. Los baremos centiles consisten en asignar a cada posible puntuación directa un valor (en una escala de 1 a 100) que se denomina centil (o percentil) y que indican el porcentaje de sujetos del grupo normativo que obtienen puntuaciones iguales 0 inferiores a las correspondientes directas. 
El modo de cálculo del centil asociado a una puntuación se resume en los siguientes pasos:

1. Disponer en una columna, ordenadas de mayor a menor o de menor a mayor, las posibles puntuaciones directas (Xi) que se puedan obtener en el test.

2. Asignar a cada puntuación su frecuencia (fi), es decir, el $n^{0}$ de sujetos del grupo normativo que la han obtenido.

3. Disponer una tercera columna de frecuencias acumuladas ( $\mathrm{Fi})$.

4. Para cada valor de $\mathrm{Fi}$, obtener el valor $\mathrm{Ci}=(100)$ $\mathrm{Fi} / \mathrm{N}$, siendo $\mathrm{Ci}$ el centil asignado a la puntuación directa Xi, Fi la frecuencia acumulada correspondiente a Xi y $\mathrm{N}$ el número total de sujetos que forman el grupo normativo.

Con esto conseguimos referenciar la puntuación obtenida individual con el del resto del grupo. Así, si un sujeto obtiene en el cuestionario de ansiedad, por ejemplo, una puntuación de 20 puntos, poco sabemos sobre su nivel de ansiedad, pero si sabemos que a esa puntuación le corresponde el centil 95 , ya conocemos que este sujeto supera en ese rasgo al $95 \%$ de los sujetos utilizados para baremar el test; si el grupo normativo fuese una muestra representativa de la población general, podríamos inferir que esta persona supera en ansiedad al $95 \%$ de las personas, y que sólo un $5 \%$ de personas son más autoritarias que él.

El Maslach Burnout Inventory (MBI) es cuestionario de 22 ítems valorados

con una escala likert de frecuencia de 7 puntos. Los resultados del MBI se expresan en tres dimensiones del estrés laboral:

- CE: cansancio emocional.

- DP: despersonalización.

- RP: realización personal.

Las dos primeras (CE y DP) tienen relación directa con los niveles de estrés asistencial y la última (RP) tiene relación inversa.

Se consideran que las puntuaciones altas en los dos primeros y baja en el tercero definen el síndrome de Burn out.

Las puntuaciones de la escala se obtienen al sumar los valores de los 22 ítems. Como punto de corte se emplearon los siguientes criterios:
- En la escala CE puntuaciones de 27 o superiores serían indicativos de un alto nivel de burnout, el intervalo entre 19 y 26 corresponderían a puntuaciones intermedias siendo las puntuaciones por debajo de 19 indicativas de niveles de burnout bajos o muy bajos.

- En la escala DP puntuaciones superiores a 10 serían nivel alto, de 6 a 9 medio, y menor de 6 bajo grado de despersonalización.

- La escala RP funciona en sentido contrario a las anteriores y así de 0 a 30 puntos indicaría baja realización personal, de 34 a 39 intermedia y superior a 40 sensación de logro.

Por tanto, una persona con alto grado de estrés asistencial mostraría altas puntuaciones en CE y DP y bajas en RP. El propio cuestionario estratifica las puntuaciones formando franjas de estrés bajo, moderado 0 alto.

Posteriormente se le realizan pruebas estadísticas mediante la suite informática IBM SPSS versión 22 de los resultados con las variables estudiadas (edad, sexo, categoría profesional y el tiempo trabajado).

\section{Resultados}

De 22 personas que trabajan en la unidad, solo 17 completaron los cuestionarios $(77,27 \%)$.

Se excluyó a una enfermera por trabajar esporádicamente en el servicio al formar parte de la plantilla volante, quedando la muestra en total en 11 enfermeras y 5 TCAE.

De las 16 personas, solo una era un hombre, por lo que no se ha hecho distinción entre sexos en los resultados para evitar sesgos.

Las edades están comprendidas entre 28 y 62 años y su media es de 44,31 $\pm 10,58$ años.

El tiempo medio que llevan trabajando en el servicio de HD es de $11,10 \pm 1,32$ años.

Con respecto al estado civil, 3 eran solteras, 1 era divorciada con pareja y el resto casados. 


\section{TEST MBI:}

En el test se observa:

- Subescala de agotamiento personal (CE):

El $12.5 \%$ tenía niveles muy altos de agotamiento, el $6.25 \%$ niveles medios y el resto niveles bajos. Al relacionar estos resultados con la variable categoría profesional, encontramos diferencia estadísticamente significativa ( $t$ de Student $p=0.047$ ), siendo las enfermeras las que tienen mayores puntaciones en CE.

- Subescala de despersonalización (DP):

El $12.5 \%$ tenía niveles muy altos de despersonalización, el 25\% tenía niveles medios y el $62.5 \%$ tenía niveles bajos. Al relacionar estos resultados con la variable categoría profesional, encontramos que no hay diferencia estadísticamente significativa (t de Student $p=0.34$ ), entre enfermeras y las TCAE.

- Subescala de realización personal (RP):

El 25\% tenía niveles bajos de realización personal, el $31.25 \%$ tenía niveles medios y el $44.75 \%$ tenía niveles altos de realización personal en el puesto de trabajo. En relación a la categoría profesional, encontramos que hay diferencia estadísticamente significativa ( $t$ de Student $p=0.015$ ), entre enfermeras y las TCAE, siendo las enfermeras las que sienten más realizadas en el trabajo.

Solo uno de los profesionales valorados presentó niveles altos de CE y DP y nivel bajo en RP, lo que indica que tiene síndrome de burn out.

Si relacionamos las puntuaciones de las tres subescalas del MBI con el tiempo de trabajo en la unidad, estratificados en cuatro grupos (< laño, de 1 a 5 años, de 6 a 10 años y más de 10 años), observamos que no hay diferencias estadísticamente significativas con ninguno de estos periodos.

$Y$ al relacionar con la edad de los participantes distribuidos en cinco grupos ( $>30$ años, 31-40, 41-50, 51-60 $\mathrm{y}>$ de 60 años), tampoco observamos diferencias significativas con ninguno de estos tramos de edad.

\section{TEST STAI:}

La media de puntuaciones para la escala Ansiedad Estado (STAI-S) fue de 19.53 y la desviación estándar de 3.02. La media para la escala Ansiedad Rasgo (STAI-T) fue de 14,24 y la desviación estándar de 7,12.
Los resultados obtenidos en ambas escalas según las franjas de edad en las que se han distribuido los participantes se muestran en la tabla 1.

Tabla 1. Resultados Test STAI según franjas de edad.

\begin{tabular}{l|l|l|}
\hline GRUPOS EDAD & STAI-S & STAI-T \\
\hline Hasta 30 años & $22 \pm 1$ & $15.33 \pm 5.51$ \\
\hline De 31- 40 años & $18,25 \pm 4,72$ & $14.75 \pm 8,3$ \\
\hline De 41- 50 años & $19,8 \pm 1,79$ & $9,2 \pm 3,56$ \\
\hline De 51- 60 años & $19 \pm 4$ & $16 \pm 8,19$ \\
\hline Mayores de 60 años & $18,5 \pm 2,12$ & $21,5 \pm 10,6$ \\
\hline
\end{tabular}

Los resultados obtenidos en ambas escalas según la categoría profesional a la que pertenecen los participantes se muestran en la figura $\mathbf{l}$.

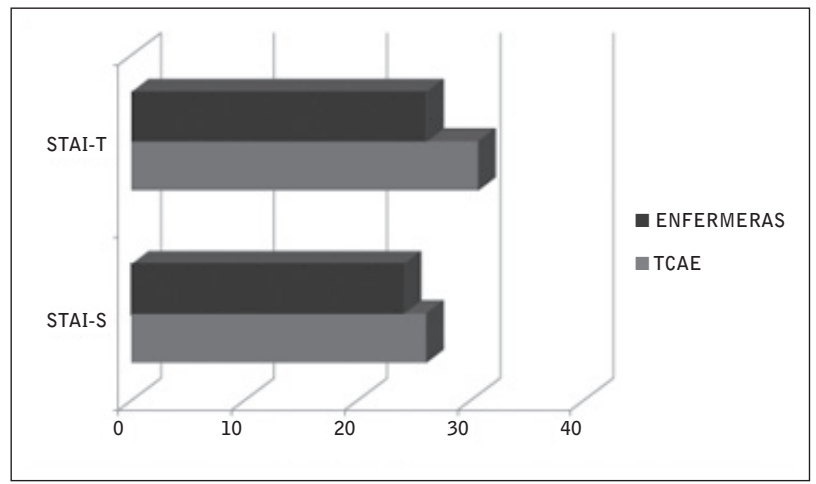

Figura 1. Comparación enfermeras y TCAE.

El coeficiente alfa de Cronbach para STAI-S por toda la muestra fue de 0.845 y del STA-T fue de 0.802 . Esto nos indica que tiene buen nivel de fiabilidad ya que la consistencia interna en ambas escalas es correcta.

\section{Discusión y conclusiones}

La participación por parte del personal de enfermería y de las TCAE del servicio de nefrología en nuestro estudio ha sido del $77,27 \%$, siendo llamativo el elevado grado de participación ya que, según la bibliografía consultada, citan la escasa participación de los profesionales en estos estudios como una debilidad metodológica argumentando causas de falta de motivación y confianza en la incidencia que puede tener un estudio de tales características ${ }^{9,10,11}$. 
Los resultados de nuestro estudio demuestran que los profesionales del servicio de nefrología evaluados mediante el Maslach Burnout Inventory, en general presentan bajos niveles de despersonalización y agotamiento personal, lo que está en la línea con otros estudios similares ${ }^{12}$.

Cabe destacar que de todo el personal evaluado solamente una persona tenía todas las características que lo definirían bajo el síndrome de burn out.

Si comparamos los resultados del test MBI de las enfermeras en relación con las TCAE, se puede observar que las enfermeras tienen más agotamiento personal en el trato con el paciente que las TCAE, pero se sienten más realizadas en el trabajo que éstas, pudiendo esto último deberse al sobreesfuerzo que las TCAE deben realizar para la movilización de pacientes y la manipulación de fluidos corporales que ocasionan fatiga física y mental ${ }^{13}$.

Además en esta muestra el tiempo que llevan trabajando en el servicio o la edad no parece influir en la presencia del Burnout.

Si tenemos en cuenta los resultados del cuestionario STAI, no hay diferencias destacables entre las enfermeras y TCAE, ni según la edad, ni según el tiempo en el servicio.

Sin embargo, destaca el aumento de la subescala Ansiedad-Rasgo con respecto a ansiedad-estado a partir de los 60 años, es decir, que aumentaría el estado en el cual la ansiedad hace ver las situaciones que ocurren en el puesto de trabajo como amenazadoras, coincidiendo nuestros resultados con otros autores ${ }^{14,15,16}$, que afirman que conforme la edad aumenta se produce una mayor tensión emocional.

Somos conscientes de las limitaciones de este estudio por el reducido tamaño de la muestra, y consideramos que sería recomendable realizar estudios más amplios para confirmar nuestros resultados.

Recibido: 19 noviembre 15

Revisado: 25 noviembre 15

Modificado: 15 diciembre 15

Aceptado: 11 enero 16

\section{Bibliografía}

1. Fornés J. Respuesta emocional al estrés laboral. Rev ROL Enf 1994;(186):31-39.

2. Más Pons Rosa, Escribà Agüir Vicenta. La versión castellana de la escala "the nursing stress scale". Proceso de adaptación transcultural. Rev. Esp. Salud Pública [revista en la Internet]. 1998 Nov [citado 2016 Abr 08] ; 72(6): 529538. Disponible en: http://scielo.isciii.es/ scielo.php?script=sci_arttext\&pid=S1135$57271998000600006 \&$ Ing $=e s$

3. Ruiz, C. 0., \& Ríos, F. L. (2004). El burnout 0 síndrome de estar quemado en los profesionales sanitarios: revisión y perspectivas. Int J Clin Health Psychol, 4(1), 137-60.

4. Maslach, C. (1982): MBI. Maslach Burnout Inventory. Palo Alto, CA: Consulting Psychologists Press.

5. Barrios Araya, Silvia, Arechabala Mantuliz, $M^{a}$ Cecilia, Valenzuela Parada, Victoria. Relación entre carga laboral y burnout en enfermeras de unidades de diálisis. Enferm Nefrol [revista en la Internet]. 2012 Mar [citado 2016 Abr 08]; 15(1): 46-55. Disponible en: http://scielo.isciii. es/scielo.php?script=sci_arttext\&pid=S225428842012000100007\&lng=es.http://dx.doi. org/10.4321/S2254-28842012000100007

6. Fernández Guzmán Isabel, Fernández Márquez $M^{a}$ Carmen, Rabadán Anta Rafael, Navalón Vila Conrado, Martínez Espejo $\mathrm{M}^{\mathrm{a}}$ Dolores. ¿Existe el síndrome de burnout en los profesionales de la salud de las unidades de hemodiálisis de la región de Murcia?. Enferm Nefrol [revista en la Internet]. 2012 Mar [citado 2016 Abr 08]; 15(1): 07-13. Disponible en: http://scielo.isciii. es/scielo.php?script=sci_arttext\&pid=S2254$28842012000100002 \& \operatorname{lng}=\mathrm{es}$. http://dx.doi. org/10.4321/S2254-28842012000100002

7. Virella, B., Arbona, C. y Novy, D. M. Psychometric properties and factor structure of the Spanish version of the State-Trait Anxiety Inventory. Journal of Personality Assessment, 1994; (63):401-412.

8. Spielberger, C. D., Gorsuch, R. L. y Lushene, R. E. STAI. Cuestionario de ansiedad estado-rasgo ( $7^{a}$ ed. rev.). Madrid (2008): TEA. 
9. Meseguer A, González R, Calatayud E. Enfermería Burnout y unidad es de alto riesgo. Enferm Clínica, $1996 ;(6): 111-24$.

10. Gil-Monte PR, Peiró JM, Valcárcel P. Influencia de las variables de carácter sociodemográfico sobre el síndrome de Burnout: un estudio en una muestra de profesionales de enfermería. Revista de Psicología Social Aplicada, 1996;6(2):43-63.

11. García M. Burnout en profesionales de enfermería de centros hosptalarios. Revista de Psicología del trabajo y las organizaciones,1991;7(18):3-12.

12. Martín MJ, Viñas J, Pérez J, Luque $M$, Soler PA. Burnotu en personal de enfermería. Rev Psiquiatría Facultad de Medicina de Barna, 1997; 24(5):113-8.

13. Mesa E, Gálvez A, Franch B, Gómez C, García JA, Vázquez $M D$, et al. Condiciones, riesgos laborales y medidas correctoras del puesto de trabajo en los enfermeros de diálisis. Comunicaciones XXVI Congreso Nacional de la Sociedad Española de Enfermería Nefrológica. 2006 Sept; 26:160-166.
14. Faura $T$, Roigé $M$, Serra $R$, Ortega $C$, Monegal $M$, Roigé $A$, et al. Prevalencia del Síndrome de Burnout en enfermeras de hospitalización y de Atención Primaria. Enferm Clínica, 1995;(5):105-10.

15. Romá MT, Ribera D, Cartagena E, Sans MI, Reig A. Estrés laboral en enfermería infantil. Una comparación con los profesionales que cuidan la población no infantil. Enferm Clínica, 1993;3 (5):13-9.

16. Atance Martínez JC. Algunas aproximaciones al Síndrome de Burnout en profesionales sanitarios. Medicina del Trabajo, 1996; (5):337-43. 